\title{
An invariant upperbound for the GNSS bootstrapped ambiguity success-rate
}

\author{
P.J.G. Teunissen \\ Department of Mathematical Geodesy and Positioning, Delft University of Technology, Kluyverweg 1, 2629 HS Delft
}

Received: 2 June 2003 / Accepted: 16 June 2003

\begin{abstract}
Carrier phase ambiguity resolution is the key to fast and high precision GPS positioning. Critical in the application of ambiguity resolution is the quality of the computed integer ambiguities. Unsuccessful ambiguity resolution, when passed unnoticed, will too often lead to unacceptable errors in the positioning results. The success or failure of carrier phase ambiguity resolution can be predicted by means of the probability of correct integer estimation, also referred to as the ambiguity success-rate. Upperbounds of the success-rate can be used to decide that ambiguity resolution has become unreliable. In this contribution we prove an upperbound for the bootstrapped success-rate. The upperbound is easy to compute and it is invariant for the class of admissible ambiguity transformations.
\end{abstract}

Keywords GNSS, integer bootstrapping, ADOP, ambiguity success-rate

\section{Introduction}

There are many ways of computing an integer ambiguity vector $\check{a} \in Z^{n}$ from its real-valued counterpart $\hat{a} \in R^{n}$, also referred to as the 'aoat' solution. To each such method belongs a mapping $S: R^{n} \mapsto Z^{n}$ from the $n$-dimensional space of real numbers to the $n$-dimensional space of integers. Due to the discrete nature of $Z^{n}$, the map $S$ will not be one-to-one, but instead a many-to-one map. This implies that different real-valued ambiguity vectors will be mapped to the same integer vector. One can therefore assign a subset $S_{z} \subset R^{n}$ to each integer vector $z \in Z^{n}$ :

$$
S_{z}=\left\{x \in R^{n} \mid z=S(x)\right\}, \quad z \in Z^{n}
$$

The subset $S_{z}$ contains all real-valued ambiguity vectors that will be mapped by $S$ to the same integer vector $z \in$
$Z^{n}$. This subset is referred to as the pull-in region of $z$. It is the region in which all ambiguity 'aoat' solutions are pulled to the same '£xed' ambiguity vector $z$. Using the pull-in regions, one can give an explicit expression for the corresponding integer ambiguity estimator. It reads $\check{a}=$ $\sum_{z \in Z^{n}} z s_{z}(\hat{a})$ with the indicator function $s_{z}(\hat{a})$ equal to one if $\hat{a} \in S_{z}$ and zero otherwise.

Since the pull-in regions defne the integer estimator completely, one can defne classes of integer estimators by imposing various conditions on the pull-in regions. One such class is referred to as the class of admissible integer estimators [Teunissen, 1999]. These integer estimators are de£ned as follows.

\section{De£nition}

The integer estimator $\check{a}=\sum_{z \in Z^{n}} z s_{z}(\hat{a})$ is said to be $a d$ missible if

$$
\begin{array}{ll}
\text { (i) } & \bigcup_{z \in Z^{n}} S_{z}=R^{n} \\
\text { (ii) } & \operatorname{Int}\left(S_{z_{1}}\right) \bigcap \operatorname{Int}\left(S_{z_{2}}\right)=\emptyset, \forall z_{1}, z_{2} \in Z^{n}, z_{1} \neq z_{2} \\
\text { (iii) } & S_{z}=z+S_{0}, \forall z \in Z^{n}
\end{array}
$$

This defnition is motivated as follows. Each one of the above three conditions describe a property of which it seems reasonable that it is possessed by an arbitrary integer ambiguity estimator. The £rst condition states that the pull-in regions should not leave any gaps and the second that they should not overlap. The absence of gaps is needed in order to be able to map any 'aoat' solution $\hat{a} \in R^{n}$ to $Z^{n}$, while the absence of overlaps is needed to guarantee that the 'ooat' solution is mapped to just one integer vector. Note that we allow the pull-in regions to have common boundaries. This is permitted if we assume to have zero probability that $\hat{a}$ lies on one of the boundaries. This will be the case when the probability density function (pdf) of $\hat{a}$ is continuous.

The third and last condition follows from the requirement that $S(x+z)=S(x)+z, \forall x \in R^{n}, z \in Z^{n}$. 
Also this condition is a reasonable one to ask for. It states that when the 'aoat' solution is perturbed by $z \in Z^{n}$, the corresponding integer solution is perturbed by the same amount. This property allows one to apply the integer remove-restore technique: $S(\hat{a}-z)+z=S(\hat{a})$. It therefore allows one to work with the fractional parts of the entries of $\hat{a}$, instead of with its complete entries. Important examples of admissible integer estimators are the estimators based on the principles of integer rounding, integer bootstrapping and integer least-squares. In this contribution we will focus on the principle of integer bootstrapping.

With the division of $R^{n}$ into mutually exclusive pull-in regions, we are in the position to consider the distribution of $\check{a}$. This distribution is of the discrete type and it will be denoted as $P(\check{a}=z)$. It is a probability mass function, having zero masses at nongrid points and nonzero masses at some or all grid points. If we denote the continuous probability density function of $\hat{a}$ as $p_{\hat{a}}(x)$, the distribution of $\check{a}$ follows as

$$
P(\check{a}=z)=\int_{S_{z}} p_{\hat{a}}(x) d x, z \in Z^{n}
$$

Note that the dependence on the chosen integer estimation principle enters through the pull-in regions $S_{z}$. The above expression holds for any distribution the 'aoat' ambiguities $\hat{a}$ might have. In most GNSS applications however, one assumes the vector of observables to be normally distributed. The estimator $\hat{a}$ is then normally distributed too, with mean $a \in Z^{n}$ and vc-matrix $Q_{\hat{a}}$. Its probability density function (pdf) reads

$$
p_{\hat{a}}(x)=\frac{1}{\sqrt{\operatorname{det}\left(Q_{\hat{a}}\right)}(2 \pi)^{\frac{1}{2} n}} \exp \left\{-\frac{1}{2}\|x-a\|_{Q_{\hat{a}}}^{2}\right\}
$$

with the squared weighted norm $\|\cdot\|_{Q_{\hat{a}}}^{2}=(.)^{T} Q_{\hat{a}}^{-1}($.$) .$

The probability $P(\check{a}=a)$ equals the probability of correct integer ambiguity estimation, the ambiguity successrate. In this contribution we will concentrate on the principle of bootstrapping and derive an invariant upperbound for its success-rate.

\section{Integer Bootstrapping}

The bootstrapped estimator follows from a sequential conditional least- squares adjustment and it is computed as follows. If $n$ ambiguities are available, one starts with the frst ambiguity $\hat{a}_{1}$, and rounds its value to the nearest integer. Having obtained the integer value of this frst ambiguity, the real-valued estimates of all remaining ambiguities are then corrected by virtue of their correlation with the £rst ambiguity. Then the second, but now corrected, realvalued ambiguity estimate is rounded to its nearest integer. Having obtained the integer value of the second ambiguity, the real-valued estimates of all remaining $n-2$ ambiguities are then again corrected, but now by virtue of their correlation with the second ambiguity. This process is continued until all ambiguities are considered. The components of the bootstrapped estimator $\check{a}_{B}$ are given as

$$
\begin{aligned}
\check{a}_{B, 1} & =\left[\hat{a}_{1}\right] \\
\check{a}_{B, 2} & =\left[\hat{a}_{2 \mid 1}\right]=\left[\hat{a}_{2}-\sigma_{21} \sigma_{1}^{-2}\left(\hat{a}_{1}-\check{a}_{B, 1}\right)\right] \\
& \vdots \\
\check{a}_{B, n} & =\left[\hat{a}_{n \mid N}\right]=\left[\hat{a}_{n}-\sum_{j=1}^{n-1} \sigma_{n, j \mid J} \sigma_{j \mid J}^{-2}\left(\hat{a}_{j \mid J}-\check{a}_{B, j}\right)\right]
\end{aligned}
$$

where the shorthand notation $\hat{a}_{i \mid I}$ stands for the $i$ th leastsquares ambiguity obtained through a conditioning on the previous $I=\{1, \ldots,(i-1)\}$ sequentially rounded ambiguities, '[.]' denotes the operation of integer rounding, $\sigma_{i, j \mid J}$ denotes the covariance between $\hat{a}_{i}$ and $\hat{a}_{j \mid J}$, and $\sigma_{j \mid J}^{2}$ denotes variance of $\hat{a}_{j \mid J}$. For a review of the theory of integer bootstrapping we refer to [Teunissen, 2001].

The bootstrapped estimator is admissible. The frst two conditions of the de£nition are satis£ed, since - apart from ties in rounding - any 'aoat' solution gets mapped to a unique integer ambiguity vector. Also the third condition of the defnition applies. To see this, let $\check{a}_{B}^{\prime}$ be the bootstrapped estimator which corresponds with $\hat{a}^{\prime}=\hat{a}-z$. It follows then from (4) that $\check{a}_{B}=\check{a}_{B}^{\prime}+z$.

The real-valued sequential conditional least-squares solution can be obtained by means of the triangular decomposition of the ambiguity variance-covariance matrix. Let the triangular decomposition of the variance-covariance matrix be given as $Q_{\hat{a}}=L D L^{T}$, with $L$ a unit lower triangular matrix and $D$ a diagonal matrix. Then $(\hat{a}-z)=$ $L\left(\hat{a}^{c}-z\right)$, where $\hat{a}^{c}$ denotes the conditional least-squares solution obtained from a sequential conditioning on the entries of $z$. The variance-covariance matrix of $\hat{a}^{c}$ is given by the diagonal matrix $D$. This shows, when a componentwise rounding is applied to $\hat{a}^{c}$, that $z$ is the integer solution of the bootstrapped method. Thus $\check{a}_{B}$ satis£es $\left[L^{-1}\left(\hat{a}-\check{a}_{B}\right)\right]=0$. Hence, if $c_{i}$ denotes the $i$ th canonical unit vector having a 1 as its $i$ th entry, the bootstrapped pull-in regions $S_{B, z}$ follow as

$$
\begin{aligned}
S_{B, z}=\left\{x \in R^{n}|| c_{i}^{T} L^{-1}(x-z) \mid \leq \frac{1}{2}\right. & \\
& i=1, \ldots, n\}, \forall z \in Z^{n}
\end{aligned}
$$

When using the bootstrapped pull-in region for the probability mass function (2) with the pdf (3), the success-rate of integer bootstrapping can be shown to follow as [Teunissen, 1998]

$$
P\left(\check{a}_{B}=a\right)=\prod_{i=1}^{n}\left(2 \Phi\left(\frac{1}{2 \sigma_{i \mid I}}\right)-1\right)
$$

where the function $\Phi$ is defned as $\Phi(x)=$ $\int_{-\infty}^{x} \frac{1}{\sqrt{2 \pi}} \exp \left\{-\frac{1}{2} v^{2}\right\} d v$. This shows that the bootstrapped success-rate is determined by the conditional 
variances $\sigma_{i \mid I}^{2}, i=1, \ldots, n$. They are the entries of the diagonal matrix $D$ of $Q_{\hat{a}}=L D L^{T}$.

The outcome of integer bootstrapping and its successrate depends on the chosen ambiguity parametrization. For instance, a simple reordering of the ambiguities will already affect the success-rate. The fact that the bootstrapped success-rate will not remain invariant when an arbitrary ambiguity transformation is applied is a consequence of the uniqueness of the triangular decomposition of $Q_{\hat{a}}$. The bootstrapped success-rate will change since the diagonal matrix $D$ of the triangular decomposition changes when an arbitrary ambiguity transformation is applied. This lack of invariance of the bootstrapped success-rate implies that one can try to improve the performance of bootstrapping by choosing an appropriate ambiguity parametrization. Since integer least- squares is known to be optimal and since bootstrapping becomes identical to integer leastsquares when the variance-covarianec matrix is diagonal, one should aim at reducing the correlations between the ambiguities. This is possible when using the decorrelating ambiguity transformation of the LAMBDA method [ $\mathrm{Teu}$ nissen, 1995]. For more information on the LAMBDA method and its applications, we refer to e.g. [Teunissen, 1993], [Tiberius and de Jonge, 1995], [Han, 1995], [de Jonge et al., 1996], [Boon and Ambrosius, 1997], [Boon et al., 1997], [Cox and Brading, 1999], [Peng et al., 1999].

Although the bootstrapped success-rate (6) is easy to compute for a particular ambiguity parametrization ones the corresponding conditional variances are given, it would still be very helpful if one could come up with an easy-tocompute invariant bound. Such a single bound needs then to be computed only once and it would cover all possible ambiguity parametrizations. In Section 4 we present an invariant upperbound of the bootstrapped success-rate. As this bound turns out to be driven by the ambiguity dilution of precision (ADOP) we £rst present some properties of the ADOP.

\section{The ambiguity dilution of precision}

The scalar ambiguity dilution of precision (ADOP) was introduced in [Teunissen, 1997a] as

$$
\mathrm{ADOP}={\sqrt{\operatorname{det} Q_{\hat{a}}}}^{\frac{1}{n}} \quad(\text { cycle })
$$

The ADOP is invariant for the class of admissible ambiguity transformations. An ambiguity transformation $\hat{z}=Z \hat{a}$ is said to be admissible if and only if all the entries of matrix $Z$ and its inverse are integer. These two conditions are needed in order to retain the integer nature of the ambiguities. It can be shown that the determinant of admissible ambiguity transformations always equals \pm 1 . We therefore have $\operatorname{det}\left(Q_{\hat{z}}\right)=\operatorname{det}\left(Z Q_{\hat{a}} Z^{T}\right)$, which shows the invariance of the ADOP. Thus the same ADOP-value is obtained, irrespective of which satellite is chosen as reference in the DD defnition of the ambiguities. Likewise, the same ADOP-value is also obtained when one uses, instead of the original variance matrix, the variance matrix of the transformed ambiguities, as produced by the LAMBDA method.

Different approaches can be used for computing the ADOP. First, one may use the variance-covariance matrix of the original DD ambiguities or of any transformed set of ambiguities. Second, for computing the determinant, one may use eigenvalues, conditional variances or, if applicable, the analytical closed form expressions as given in [Teunissen, 1997b].

When the eigenvalues $\lambda_{\hat{a}_{i}}$ of the ambiguity variance matrix are used, we have

$$
\mathrm{ADOP}=\prod_{i=1}^{n} \lambda_{\hat{a}_{i}}^{\frac{1}{2 n}}
$$

Instead of working with eigenvalues, a cheaper way would be to make use of the conditional variances. This approach is based on using a triangular decomposition or a Cholesky decomposition of the ambiguity variance matrix or its inverse. The entries of the diagonal matrix $D$ in the $L D L^{T}$ decomposition of the variance matrix are the sequential conditional variances of the ambiguities. Since the determinant of the diagonal matrix $D$ equals the determinant of the variance matrix, the ADOP becomes

$$
\mathrm{ADOP}=\prod_{i=1}^{n} \sigma_{\hat{a}_{i \mid I}}^{\frac{1}{n}}
$$

In case of bootstrapping the conditional variances $\sigma_{\hat{a}_{i \mid I}}^{2}$ are usually already available.

When the ambiguities are completely decorrelated, the ADOP equals the geometric mean of the standard deviations of the ambiguities. This follows from $\operatorname{det}\left(Q_{\hat{a}}\right)=$ $\prod_{i=1}^{n} \sigma_{\hat{a}_{i}}^{2} \operatorname{det}\left(R_{\hat{a}}\right)$, where $R_{\hat{a}}$ is the ambiguity correlation matrix. Since the decorrelating ambiguity transformation of the LAMBDA method produces ambiguities that are largely decorrelated, the ADOP approximates the average precision of these transformed ambiguities.

\section{The invariant upperbound and its proof}

We now come to the main result of this contribution. As the following theorem shows, the invariant ADOP can be used to obtain an upperbound for the bootstrapped successrates.

\section{Theorem (Invariant upperbound)}

For any admissible ambiguity parametrization the bootstrapped succes- rate can be bounded from above as

$$
\begin{aligned}
P\left(\check{a}_{B}=a\right) & =\prod_{i=1}^{n}\left(2 \Phi\left(\frac{1}{2 \sigma_{i \mid I}}\right)-1\right) \\
& \leq\left(2 \Phi\left(\frac{1}{2 \mathrm{ADOP}}\right)-1\right)^{n}
\end{aligned}
$$


Note that the upperbound is sharp in the sense that it will be reached when all ambiguities are completely decorrelated. The above easy-to- compute upperbound can be used to decide on the potential usefulness of bootstrapping for ambiguity resolution. If in any application the upperbound turns out be too small the conclusion must be that one can not expect carrier phase ambiguity resolution to be successful when it is based on the principle of integer bootstrapping.

The above upperbound was introduced without explicit proof in [Teunissen, 1998]. We will now give its proof. The proof is rather lengthy and will therefore be given in a number of steps. For the proof we also make use of two important results which are given in the Appendix.

Step 1: The above theorem will be proven by solving the maximization problem

$$
\max _{x_{i}} \prod_{i=1}^{n} F\left(x_{i}\right) \text { subject to } \prod_{i=1}^{n} x_{i}=c
$$

where $F(x)=2 \Phi(x)-1, x_{i}=1 /\left(2 \sigma_{i \mid I}\right)>0$ and $c$ is a known constant. The constraint has been included to reaect the fact that the product of the ambiguity conditional variances, $\prod_{i=1}^{n} \sigma_{i \mid I}^{2}$, is invariant for any arbitrary admissible ambiguity transformation. The constant is therefore given as

$$
c=\left(\frac{1}{2 \mathrm{ADOP}}\right)^{n}
$$

In order to make the above maximization problem more manageable we transform it such that the constraint becomes linear and the objective function can be written as a sum instead of as a product. For that purpose we take the logarithm of the objective function, the logarithm of the constraint and reparametrize by replacing the parameters $x_{i}$ with $x_{i}=\exp v_{i}$. As a result we get the maximization problem

$$
\max _{v_{i}} \sum_{i=1}^{n} \ln F\left(\exp v_{i}\right) \text { subject to } \sum_{i=1}^{n} v_{i}=\ln c
$$

We may now think of applying the optimization theorem as given in the Appendix (note: maximizing an objective function is equivalent to minimizing -1 times the objective function). For the theorem to be applicable we need to show that the objective function $\sum_{i=1}^{n} \ln F\left(\exp v_{i}\right)$ is concave (or $\sum_{i=1}^{n}-\ln F\left(\exp v_{i}\right)$ is convex) with continuous frst derivatives and that the feasible set of the constraint is convex. The latter is easily shown since the constraint is linear. It is also easily shown that the frst order derivative of $F(x)$ is continuous. This leaves us to show that the objective function is concave. But since the sum of concave functions is again concave, it suffces to show that

$\ln F(\exp v)$ is concave
Step 2 We will now use Prekopa's theorem of the Appendix to show that (14) is indeed true. Since $F(x)=$ $2 \Phi(x)-1=P\left(u \leq x^{2}\right)$, with $u$ distributed as a central Chi-squared distribution with one degree of freedom, $u \sim$ $\chi^{2}(1,0)$, we have $F(\exp v)=P\left(\frac{1}{2} \ln u \leq v\right)$. We therefore need to show that $\ln F(\exp v)=\ln P\left(\frac{1}{2} \ln u \leq v\right)$ is concave, or that

$$
\begin{array}{r}
\ln P\left(\frac{1}{2} \ln u \leq \alpha v+(1-\alpha) w\right) \geq \\
\alpha \ln P\left(\frac{1}{2} \ln u \leq v\right)+(1-\alpha) \ln P\left(\frac{1}{2} \ln u \leq w\right) \\
\forall \alpha \in[0,1]
\end{array}
$$

According to Prekopa's theorem this is true when

$$
\begin{aligned}
& \ln p_{\frac{1}{2} \ln u}(\alpha v+(1-\alpha) w) \geq \\
& \quad \alpha \ln p_{\frac{1}{2} \ln u}(v)+(1-\alpha) \ln p_{\frac{1}{2} \ln u}(w) \forall \alpha \in[0,1]
\end{aligned}
$$

with $p_{\frac{1}{2} \ln u}(v)$ the pdf of $\frac{1}{2} \ln u$. In order to verify (15) we need to determine this pdf. Since $u \sim \chi^{2}(1,0)$, its pdf is given as $p_{u}(x)=\left(\sqrt{2} \Gamma\left(\frac{1}{2}\right)\right)^{-1} x^{-\frac{1}{2}} \exp -\frac{1}{2} x, 0<x<$ $\infty$. Using the transformation $y=\frac{1}{2} \ln x$ the pdf of $\frac{1}{2} \ln u$ follows as

$$
\begin{aligned}
p_{\frac{1}{2} \ln u}(y) & =\frac{p_{u}(\exp 2 y)}{\frac{1}{2} \exp -2 y} \\
& =2\left(\sqrt{2} \Gamma\left(\frac{1}{2}\right)\right)^{-1} \exp y \exp \left\{-\frac{1}{2} \exp 2 y\right\}
\end{aligned}
$$

We therefore have

$$
\ln p_{\frac{1}{2} \ln u}(y)=\ln \left(2\left(\sqrt{2} \Gamma\left(\frac{1}{2}\right)\right)^{-1}\right)+y-\frac{1}{2} \exp 2 y
$$

which is easily shown to be concave. Therefore (15) is true and by Prekopa's theorem also (14) is true. The conclusion reads therefore that all conditions for the optimization theorem to be applicable are satisfed.

Step 3 Application of the optimization theorem as given in the Appendix boils down to fnding the solution of $\partial_{v_{i}} L\left(v_{i}, \lambda\right)=0, i=1, \ldots, n$, with the Lagrangian

$$
L\left(v_{i}, \lambda\right)=\sum_{i=1}^{n} \ln F\left(\exp v_{i}\right)+\lambda\left(\sum_{i=1}^{n} v_{i}-\ln c\right)
$$

This gives

$$
\begin{aligned}
\partial_{v_{i}} L\left(v_{i}, \lambda\right)=\frac{F^{\prime}\left(\exp v_{i}\right)}{F\left(\exp v_{i}\right)} \exp v_{i}+\lambda & =0, \\
\text { for } i & =1, \ldots, n
\end{aligned}
$$

in which $F^{\prime}$ denotes the frst derivative of $F$. These $n$ equations are satisfed when all $v_{i}$ are equal. And since 
they also need to satisfy the constraint $\sum_{i=1}^{n} v_{i}=\ln c$, it follows that $v_{i}=\frac{1}{n} \ln c$, or in terms of the original parameters, that $x_{i}=c^{1 / n}$. Together with (12) this fnally gives

$$
x_{i}=\frac{1}{2 \mathrm{ADOP}}, \text { for } i=1, \ldots, n
$$

We have therefore proven that $\prod_{i=1}^{n} F\left(\frac{1}{2 \mathrm{ADOP}}\right)$ is the solution of (11). This concludes the proof of (10).

\section{References}

Boon, F., B. Ambrosius (1997) Results of real-time applications of the LAMBDA method in GPS based aircraft landings, Proceedings KIS97, pp. 339-345.

Boon, F., P.J. de Jonge, C.C.J.M. Tiberius (1997) Precise aircraft positioning by fast ambiguity resolution using improved troposphere modelling, Proceedings ION GPS-97, Vol. 2, pp. 1877-1884.

Chong, E.K.P., S.H. Zak (1996) An Introduction to Optimization, John Wiley and Sons.

Cox, D.B. and J.D.W. Brading (1999) Integration of LAMBDA ambiguity resolution with Kalman £lter for relative navigation of spacecraft, Proc. ION NTM-99, pp. 739-745.

de Jonge, P.J., C.C.J.M. Tiberius, P.J.G. Teunissen (1996) Computational aspects of the LAMBDA method for GPS ambiguity resolution, Proc. ION GPS-96, pp. 935-944.

Han, S. (1995) Ambiguity resolution techniques using integer least- squares estimation for rapid static or kinematic positioning, Symposium Satellite Navigation Technology: 1995 and beyond, Brisbane, Australia, 10 p.

Peng, H.M., F.R. Chang, L.S. Wang (1999) Attitude determination using GPS carrier phase and compass data, Proc ION NTM-99, pp. 727-732.

Prekopa, A. (1971) Logarithmic concave measures with applications, Acta Sci. Math. Statist., 32, 302-316.

Teunissen, P.J.G. (1993) Least-squares estimation of the integer GPS ambiguities, Invited Lecture, Section IV Theory and Methodology, IAG General Meeting, Beijing, China, August 1993. Also in: LGR Series, No. 6, Delft Geodetic Computing Centre.

Teunissen, P.J.G. (1995) The least-squares ambiguity decorrelation adjustment: a method for fast GPS integer ambiguity estimation, Journal of Geodesy, 70: 65-82.

Teunissen, P.J.G. (1997a) A canonical theory for short GPS baselines. Part IV: precision versus reliability, Journal of Geodesy, 71: 513-525.

Teunissen, P.J.G. (1997b) Closed form expressions for the volume of the GPS ambiguity search spaces, Artificial Satellites, Vol. 32, No. 1, pp. 5-20.

Teunissen, P.J.G. (1998) The success probability of integer GPS ambiguity rounding and bootstrapping, Journal of Geodesy, 72: 606- 612.

Teunissen, P.J.G. (1999) An optimality property of the integer least-squares estimator, Journal of Geodesy, 73: 587-593.
Teunissen, P.J.G. (2001) GNSS ambiguity bootstrapping: theory and application, In: Proc. KIS2001, International Symposium on Kinematic Systems in Geodesy, Geomatics and Navigation, Banff, Canada, pp. 246-254.

Tiberius, C.C.J.M., P.J. de Jonge (1995) Fast positioning using the LAMBDA method, Proceedings DSNS-95, Paper 80, $8 \mathrm{p}$.

\section{Appendix}

In this appendix the two theorems are given which are used in the proof of the invariant upperbound for the bootstrapped success rate.

Theorem (global optimization)

Let $f: R^{n} \mapsto R, f \in C^{1}$, be a convex function on the set of feasible points

$$
\Omega=\left\{x \in R^{n} \mid h(x)=0\right\}
$$

where $h: R^{n} \mapsto R^{m}, h \in C^{1}$, and $\Omega$ is convex $\left(C^{1}\right.$ denotes the class of functions with continuous frst order derivatives). Suppose there exists $\hat{x} \in \Omega$ and $\hat{\lambda} \in R^{m}$ such that

$$
\partial_{x} f(\hat{x})+\hat{\lambda}^{T} \partial_{x} h(\hat{x})=0
$$

Then $\hat{x}$ is a global minimizer of $f$ over $\Omega$.

Proof: see [Chong and Zak, 1996, p. 379]

Theorem (Prekopa)

Let $p(x), x \in R^{n}$, be a density function for which

$$
\begin{array}{r}
\ln p(\alpha x+(1-\alpha) y) \geq \alpha \ln p(x)+(1-\alpha) \ln f(y), \\
\forall \alpha \in[0,1]
\end{array}
$$

Then

$$
\begin{aligned}
& \ln P(x \in \alpha A+(1-\alpha) B) \geq \\
& \alpha \ln P(x \in A)+(1-\alpha) \ln P(x \in B)
\end{aligned}
$$

where $A, B$ are any sets in $R^{n}$ and their convex combination is de£ned as

$$
\begin{aligned}
& \alpha A+(1-\alpha) B= \\
& \quad\left\{z \in R^{n} \mid z=\alpha x+(1-\alpha) y, x \in A, y \in B\right\}
\end{aligned}
$$

and $P$ denotes the probability.

Proof: see [Prekopa, 1971] 